\title{
High-speed flow of interacting organic polaritons
}

\author{
Giovanni Lerario $^{1}$, Dario Ballarini ${ }^{1}$, Antonio Fieramosca ${ }^{1}$, Alessandro Cannavale ${ }^{1,2}$, Armando Genco ${ }^{2}$, \\ Federica Mangione ${ }^{1}$, Salvatore Gambino ${ }^{1,2}$, Lorenzo Dominici ${ }^{1}$, Milena De Giorgi ${ }^{1}$, Giuseppe Gigli ${ }^{1,2}$ and \\ Daniele Sanvitto ${ }^{1}$
}

The strong coupling of an excitonic transition with an electromagnetic mode results in composite quasi-particles called exciton polaritons, which have been shown to combine the best properties of their individual components in semiconductor microcavities. However, the physics and applications of polariton flows in organic materials and at room temperature are still unexplored because of the poor photon confinement in such structures. Here, we demonstrate that polaritons formed by the hybridization of organic excitons with a Bloch surface wave are able to propagate for hundreds of microns showing remarkable third-order nonlinear interactions upon high injection density. These findings pave the way for the study of organic nonlinear light-matter fluxes and for a technologically promising route of the realization of dissipation-less on-chip polariton devices operating at room temperature.

Light: Science \& Applications (2017) 6, e16212; doi:10.1038/lsa.2016.212; published online 24 February 2017

Keywords: optical nonlinearity; organic materials; polaritons; surface waves; 2D optics

\section{INTRODUCTION}

Exciton polaritons are hybrid quasi-particles arising from the strong coupling between excitons and photons that possess the features of both of their components ${ }^{1-3}$. Photons, which are massless and noninteracting particles, reduce the exciton mass by three orders of magnitude $\mathrm{e}^{4-6}$, whereas excitons retain their nonlinear properties that are four orders of magnitude higher than those of standard nonlinear optical media ${ }^{3,7,8}$. In the last 10 years, these attributes have led to the observation of fascinating new physics in solid state systems such as polariton condensation ${ }^{9-11}$, superfluidity ${ }^{12,13}$, quantized vortices ${ }^{14,15}$ and their nonlinear dynamics ${ }^{16}$. Recently, exciton polaritons have also attracted a strong technological interest as ultrafast and dissipation-less optical devices ${ }^{17-20}$. Exciton polaritons are perfect candidates for these applications: the excitonic component makes them extremely sensitive to small power changes, whereas the photonic part allows for information manipulation with high speed, efficiency and no heat dissipation ${ }^{21-23}$.

The overwhelming majority of the previous research has focused on adopting inorganic-based systems (typically semiconductor quantum wells) operating at cryogenic temperatures; however, this limits their potential technological applications. Due to their ease of fabrication, low costs and high binding energies ${ }^{24,25}$, organic polaritons are an emerging field with an outstanding potential, as demonstrated by the recent observation of room temperature condensation ${ }^{26,27}$. Conventional polaritonic structures are planar microcavities where the optical active layer is embedded between two Distributed Bragg Reflectors (DBRs). However, the high dissipation rate of organic planar microcavities (short lifetimes) together with their relatively low group velocity (typically $1-2 \mu \mathrm{m} \mathrm{ps}^{-1}$ ) has so far prevented the observation of the fascinating physics related to the flows of light-matter particles as well as any possible applications in cascadable-on-chip technologies that have been intensively investigated in inorganic systems ${ }^{28-31}$. Here, we show organic polariton propagation for distances longer than 120 microns and with group velocities of $\sim 50 \%$ of the speed of light in a single DBR structure operating at room temperature.

To obtain such fast velocities, an organic exciton is coupled with a high-quality Bloch surface wave (BSW). The BSW is an evanescent, lossless and propagating optical mode with very high group velocity ${ }^{32-34}$, that differs from other surface modes such as the Tamm states or surface plasmons because it does not undergo dissipation caused by metallic $\operatorname{losses}^{35}$. Due to their high-quality factor, BSWs are widely studied for sensing applications, and their remarkable potential for the field of onchip optical manipulation has only recently been explored ${ }^{36-39}$. Unlike the bare optical BSW investigated in these works, the excitonic component of the Bloch surface wave polariton (BSWP) allows the exploitation of nonlinear interactions. Here, we demonstrate the blueshift of the polariton resonance with increasing particle densities, enabling the local dynamic manipulation of the BSWP dispersion by external laser pulses and also laying the groundwork for polariton devices with logic functionalities that operate at room temperature.

\section{MATERIALS AND METHODS}

The DBR fabrication process consists of the deposition of seven $\mathrm{TiO}_{2} / \mathrm{SiO}_{2}$ pairs $\left(d_{\mathrm{TiO}_{2}}=85 \mathrm{~nm}\right.$ and $\left.d_{\mathrm{SiO}_{2}}=120 \mathrm{~nm}\right)$ on a $130-\mu \mathrm{m}$ thick glass substrate using an e-beam evaporator.

${ }^{1}$ CNR NANOTEC -Istituto di Nanotecnologia, Via Monteroni, 73100 Lecce, Italy and ²Dipartimento di matematica e fisica ‘Ennio De Giorgi', Università del Salento, 73100 Lecce, Italy Correspondence: D Ballarini, Email: dario.ballarini@nanotec.cnr.it

Received 27 January 2016; revised 18 August 2016; accepted 31 August 2016; accepted article preview online 5 September 2016 
A 35-nm thick layer of a perylene derivative (Lumogen Red F305), a small molecule with the absorption and emission spectra shown in Figure 1a, is thermally evaporated onto the $\mathrm{SiO}_{2}$ top layer of the DBR, with the base pressure of $\sim 10^{-7} \mathrm{mbar}$, and at the deposition rate of $\sim 1.0 \AA \mathrm{s}^{-1}$.

The resulting structure sustains only high-quality TE polarized BSWP modes. The extraction of the signals lying beyond the critical angle is obtained through a high numerical aperture (NA 1.49) oil immersion microscope objective positioned at the glass substrate side, obviating the need to use additional optical elements ${ }^{40,41}$. The spectrometer and the CCD imaging camera (Princeton Instruments, Inc., Trenton, NJ, USA) allow the energy resolution of the BSWP signal. In the non-resonant configuration (upper part of Figure 1c), a TM-polarized continuous wave laser at $2.33 \mathrm{eV}$ is focused $(2 \mu \mathrm{m}$ spot radius) through a second objective (NA 0.45) from the organic deposition side to prevent any possible coupling of the excitation laser to the surface propagating modes. The laser intensity on the sample surface is limited to $10 \mathrm{~W} \mathrm{~cm}^{-2}$, preventing damage to the organic layer. Path A in Figure 1c shows the optical configuration used for the space-space and energy-space imaging of the emission: the back focal plane (Fourier plane) of the detection objective is recreated along the detection line where the slit S1 allows the selection of specific momentum space regions. This selection is monitored by adding a lens (path B in Figure 1c) that allows the visualization of the angular distribution of the signal. The spectrometer in path $\mathrm{B}$ is used for the energy resolution of the mode dispersion, with wave vectors obtained according to $k_{\perp}=\frac{2 \pi}{\lambda} n_{g} \sin \theta$, where $n_{g}$ is the glass substrate refractive index.

A different experimental configuration, sketched in the bottom part of Figure 1c, is used for the resonant injection of the polaritons. To match the BSWP dispersion (both in energy and momentum), the oil immersion objective is used for both the excitation and detection of the polariton mode, that is, operating in the reflection configuration. The slit S2 is positioned at the image plane of the sample and allows the selection of different areas in the field of view. A $300-\mathrm{mm}$ spectrometer with a 1200 -gg mm${ }^{-1}$ grating blazed at $600 \mathrm{~nm}$ and a high-sensitivity CCD imaging camera are used for collecting the energy-resolved images with an overall resolution of $0.4 \mathrm{~nm}$. In the non-resonant configuration, a low-pass filter at $600 \mathrm{~nm}$ is used for the energy-resolved propagation measurements to cut-off the residual laser signal passing through the sample.

The experimental data are supported by transfer matrix (TMM) calculations; the thicknesses and refractive indexes of the layers constituting the DBR and of the organic material are evaluated by ellipsometric measurements (Supplementary Information).

\section{RESULTS AND DISCUSSION}

The calculated dispersion by TMM and the experimental data of the BSWP emission under non-resonant excitation are shown in Figure 2a in the top and bottom panels, respectively. The experimental emission perfectly matches the simulated polaritonic mode (dashed orange line on the experimental emission in Figure 2a) that deviates from the bare optical BSW (blue line) when approaching the exciton energy (yellow line at $2.13 \mathrm{eV}$ ), showing the anti-crossing behavior typical of strongly coupled systems with a Rabi splitting of $186 \mathrm{meV}$. Short-propagating evanescent modes attributed to the limit of the DBR optical band-gap (sideband modes) are also visible in both the simulated and experimental results at low energies and high momenta ${ }^{42}$.

The BSWP is highly sensitive to small changes of the optical index at the surface of the device ${ }^{43}$, and any spatial inhomogeneity of the a

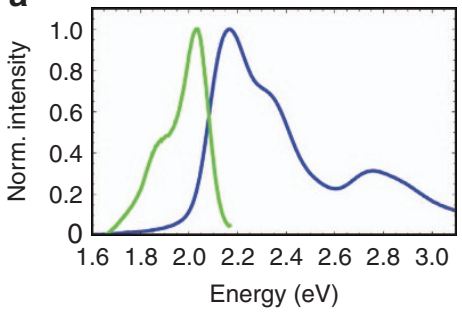

b

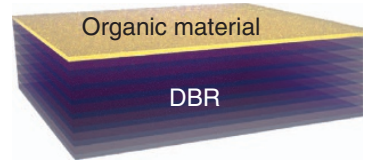

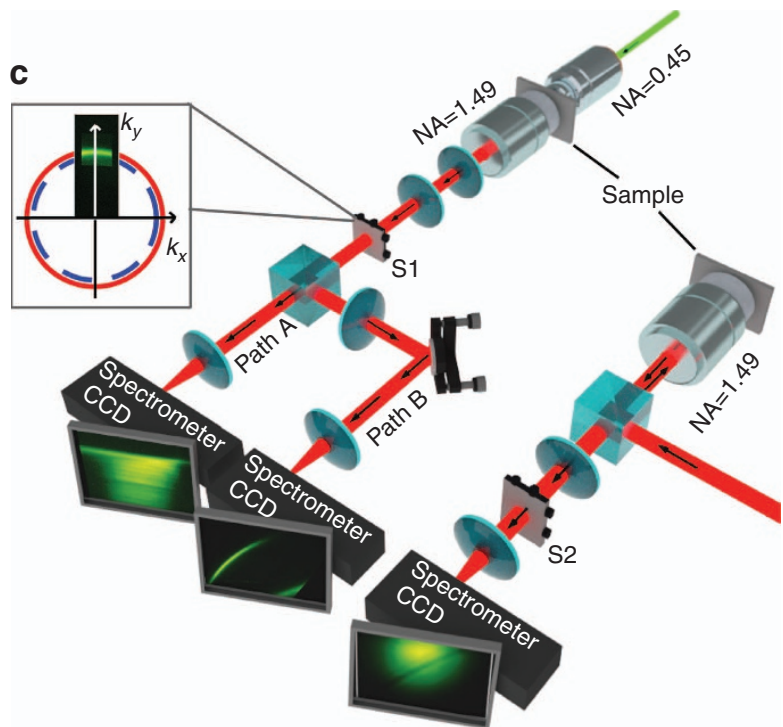

Figure 1 Material, sample structure and optical setup. (a) Absorption and emission spectra of Lumogen Red F305 in the solid state (35-nm thick thermally evaporated thin film). (b) Illustration of the sample structure. The organic layer is deposited on a DBR composed of seven pairs of dielectric oxides $\left(\mathrm{TiO}_{2} /\right.$ $\mathrm{SiO}_{2}$ ). (c) In the upper part, a sketch of the leakage radiation microscope setup in a non-resonant configuration is shown. The excitation source (at $2.33 \mathrm{eV}$ ) is focused on the sample from the organic deposition side. The emission is collected through a microscope objective with $N A=1.49$, extracting the signals lying beyond the critical angle. The use of two detection arms together with a spectrometer and a CCD imaging camera enables visualization of the energyresolved space and momentum maps. The slit (S1) are used for the selection of specific directions of propagations. Inset: experimental 2D momentum space with S1 selection, with critical angle and BSWP indicated by dashed blue and red lines, respectively. In the bottom part, a sketch of the resonant configuration is shown. The excitation laser (100-fs laser pulse) is an energetically matched with the BSWP mode and focused through the oil immersion objective. The dispersion map of the reflected light is visualized on the CCD camera. The slit (S2) are used for the selection of the excitation spot area to cutoff the propagating signal and reveal the BSWP as a dip in the reflectance map. 
a
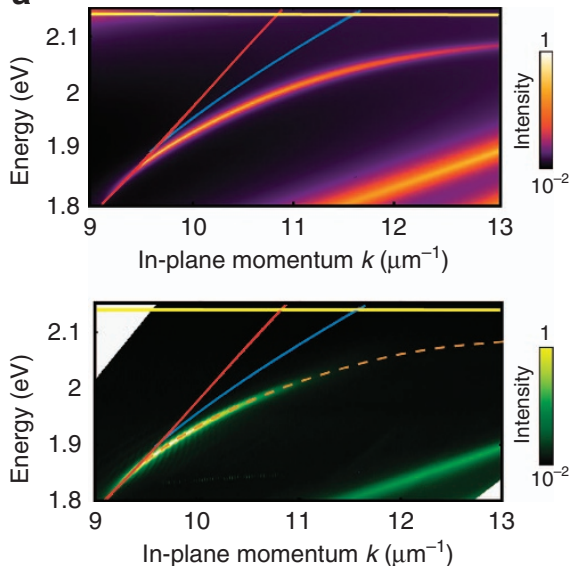

b $10^{-2}$ Intensity 1

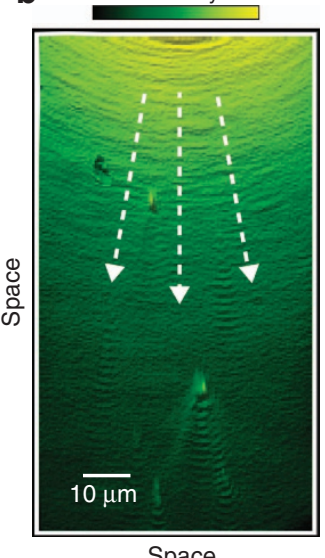

C

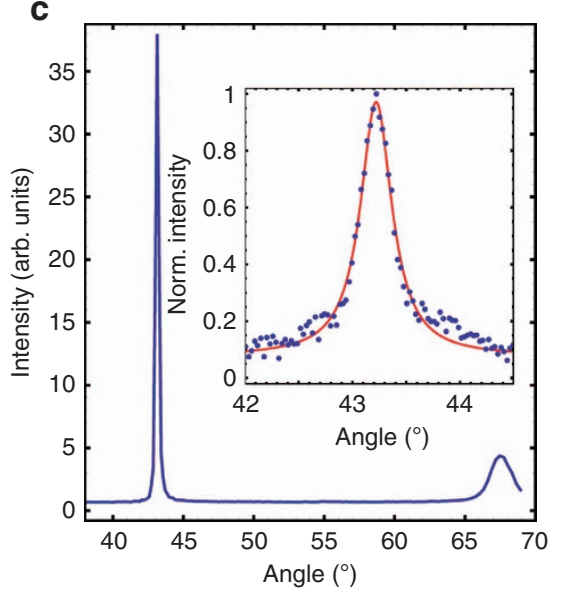

Figure 2 Dispersion and space propagation of the BSWP. (a) Top panel: TMM calculation of the electromagnetic mode dispersions. The light cone is delimited by the red line, and the blue line indicates the bare optical BSW and the yellow line indicates the exciton energy (2.13 eV). The sharp signal in the center of the image is the BSWP mode, and the signal at higher momenta corresponds to the sideband modes of the DBR stop band. Bottom panel: Experimental measurement of the emission dispersion. Theoretical BSWP (dashed orange line), obtained with all the parameters extracted from the materials constituting the as-grown structure (Supplementary Information), fits the experimental results exactly. A weaker signal from the sideband modes is also detected. (b) Space map of the non-resonant excited polariton emission; the white arrows are a guide for the eye indicating the radial directions starting from the excitation spot, that is, the BSWP directions of propagation. (c) The emission intensity profile at 1.925 eV as a function of the detection angle. In the inset, the experimental data (blue dots) are fitted with a Lorentzian function with a full width at half maximum of $0.4^{\circ}$ (red line).

a

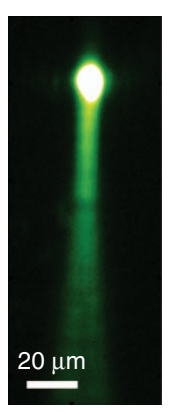

b
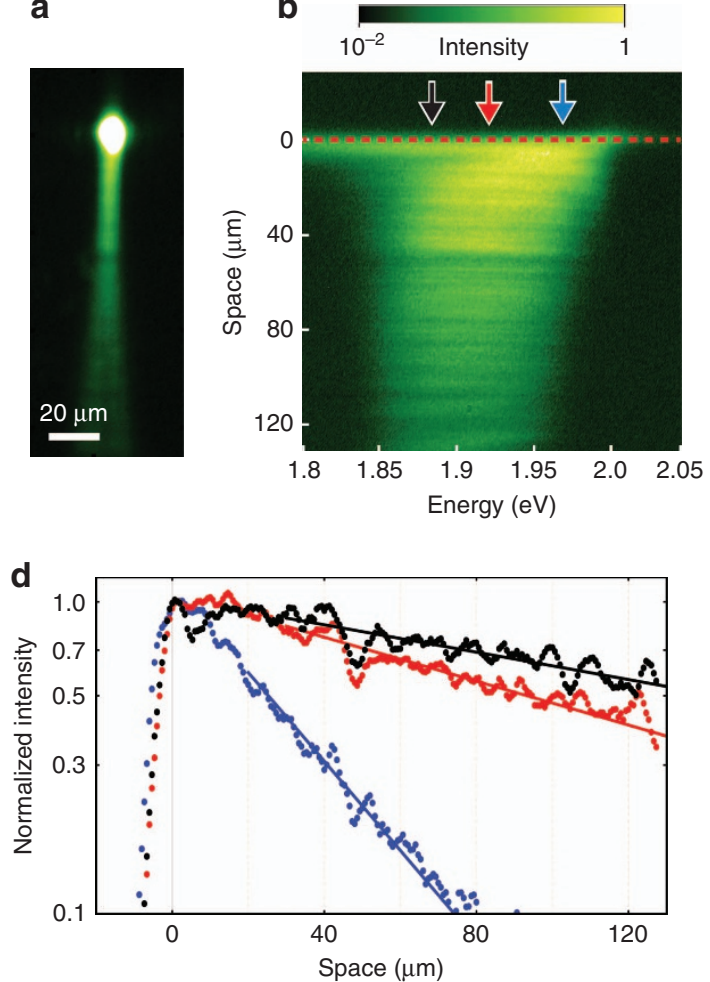

C

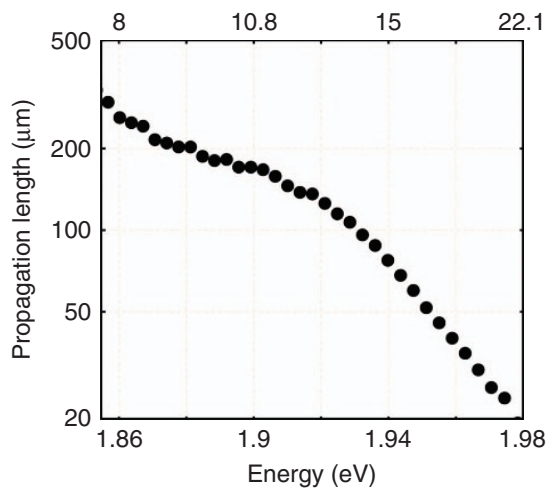

e

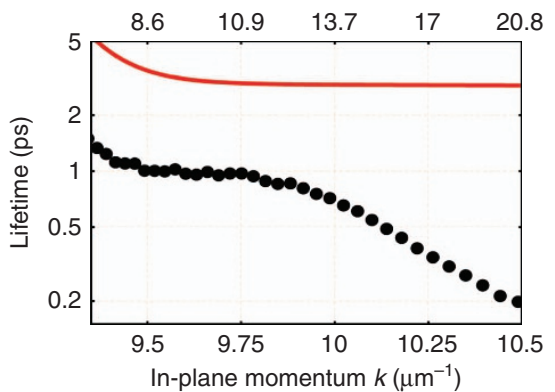

Figure 3 Polariton propagation. (a) Space propagation of the BSWP after filtering in momentum space and with a low-pass filter at $600 \mathrm{~nm}$. (b) Space propagation in the energy domain (associated to a). The red dashed line indicates the position of the excitation spot. (c) Propagation lengths versus energy and exciton fraction. (d) Intensity profiles (logarithmic scale) of the energy-resolved polariton propagation at different exciton fractions. At $1.97 \mathrm{eV}$ (blue line), with the excitonic component $>20 \%$, the propagation distance is $\sim 30 \mu \mathrm{m}$; at $1.92 \mathrm{eV}$ (red line), the exciton fraction is $12.7 \%$ and the propagation length is $120 \mathrm{~nm}$. At lower energies $(1.88 \mathrm{eV}$, black line), an initial rise of the intensity is observed, followed by a long decay of $200 \mu \mathrm{m}$. (e) Polariton lifetime, versus in-plane wavevector and exciton fraction, evaluated from propagation lengths and group velocity. The red line is the TMM calculation of the bare BSW lifetimes. 
sample deposition results in a broader BSWP dispersion ${ }^{34}$. Therefore, the sharpness of the polariton mode that is clearly visible in the emission profile shown in Figure 2c, is a first hint of the high local homogeneity of the organic deposition.

The propagation of the polariton fluid on the sample surface is clearly visible in Figure $2 \mathrm{~b}$, where a few defects in the structure act as small perturbations on the flow trajectory. This also demonstrates that BSWP is an ideal workbench for the study of transport properties of dipole-like excitations strongly coupled with an electromagnetic mode; these are currently a focus of intense investigations and have recently been proposed to be strongly enhanced in the case of molecules and atoms placed inside an optical cavity ${ }^{44-47}$.

Figure 3 a shows the polariton flow along a given direction, obtained by filtering the signal in the back focal plane of the detection objective (slit S1 in the inset of Figure 1c). A low-energy pass filter at $600 \mathrm{~nm}$ is used to filter out the non-resonant laser light, resulting in the energyresolved emission spectrum presented in Figure 3b. The residual emission of uncoupled excitons is detected close to the excitation beam position (red dashed line).

Sideband modes and short-living polaritons at higher energies populated through non-resonant pumping can also decay into the lower polariton branch; however, owing to their weak-propagating nature, they can only affect polariton formation very close to the excitation spot (less than $10 \mu \mathrm{m})^{48-50}$. Although this 'filling effect' is small, it produces the initial rise of the signal at lower energies, as shown in Figure $3 \mathrm{~b}$ and $3 \mathrm{~d}$ (black arrow). At longer distances, the emission is attributed only to the long propagating BSWP and follows an exponential decay. The propagation length values at different energies, corresponding to different excitonic fractions, are shown in Figure $3 \mathrm{c}$ and exhibit ranges up to $300 \mu \mathrm{m}$ ( $8 \%$ of exciton content), with a mean propagation length of $\sim 120 \mu \mathrm{m}$ and $13 \%$ of excitonic content.

The polariton lifetimes $\left(\tau_{\text {pol }}\right)$ can be estimated from the experimental propagation length and group velocity $\left(v_{\mathrm{g}}\right)^{51}$. The flow speed as a function of energy (E) is extracted directly from the BSWP dispersion as $v_{\mathrm{g}}=\frac{1}{\hbar} \frac{\partial E}{\partial k^{\circ}}$. Group velocities ranging from 120 to $250 \mu \mathrm{m} \mathrm{ps}^{-1}$ (Supplementary Information) are obtained, $\sim 100$ times faster than in standard planar microcavities. At the energy of the maximum emission intensity, the polariton lifetime is $\sim 1 \mathrm{ps}$ (see data in Figure 3e) and the dissipative character of the BSWP is attributed mainly to the excitonic component.

Indeed, the DBR mode quality (evaluated from the ellipsometric data of the DBR structure) is estimated to be close to $E / \Delta E=7000$, giving a corresponding photon lifetime of $\sim 3 \mathrm{ps}$ (red line in Figure 3e). Although this value is about one order of magnitude higher than that obtained in the state-of-the-art organic microcavities, it is not as high as the lifetimes reported for inorganic planar microcavities, where lifetimes of some hundreds of ps have been obtained $^{51,52}$. However, thanks to the high speed of BSWP, the propagation lengths obtained in the present structure are comparable to the ones achieved with the best inorganic-based planar microcavities $^{52,53}$.

To fully exploit the potential of this system, we resonantly injected polaritons using the configuration depicted in the bottom panel of Figure 1c. Resonant excitation below the absorption energy of the exciton is a favorable configuration for the observation of the blueshift of the BSWP induced by polariton-polariton interactions only, avoiding the formation of a large reservoir of uncoupled excitons and reducing the heating of the sample and its degradation.

By selecting the sample area under the excitation spot through the slit S2 (Figure 1c), the propagating signal can be subtracted from the energy-momentum map, and the BSWP appeared as a dip in the reflectance dispersion, as shown in Figure 4a for the low-energy density signal of $150 \mu \mathrm{J} \mathrm{cm}^{-2}$ (measured before the pump enters the microscope objective).

When the pumping energy density is increased to $\rho_{E}=10 \mathrm{~mJ} \mathrm{~cm}^{-2}$, the BSWP mode energy blueshifts. The blue (red) dots in Figure 4c represent the experimental peaks at different energies for the low (high) pumping power. The experimental dispersion at low power perfectly matches the results of the TMM calculation with the exciton peak at $2.13 \mathrm{meV}$ (blue line in Figure 4c), whereas at $\rho_{E}=10 \mathrm{~mJ} \mathrm{~cm}{ }^{-2}$ the TMM reproduces the experimental dispersion resulting from an exciton energy blueshift of $\sim 8 \mathrm{meV}$ (red line in Figure $4 \mathrm{c}$ ). Despite the reflectance spectra that are inevitably broader than the actual polariton dispersion due to the setup angular resolution $\left(0.1^{\circ}\right)$ that affects the momentum resolution $\left(0.018 \mu \mathrm{m}^{-1}\right)$, the shift of the polariton energy is larger than the mode line width and can be clearly resolved in our experiments, as shown in Figure $4 \mathrm{~b}$. This demonstrates the possibility of using polariton nonlinearities to tune a mode in and out of resonance with an external optical beam.

The wide range of energies covered by the laser pulse makes it possible to observe the trend of increasing polariton blueshift for energies approaching the exciton resonance (Figure 4d), as expected when increasing the exciton fraction of the BSWP mode.

Based on the BSWP depth in the energy-momentum map, it is reasonable to consider that a fraction of $\sim 1 \%$ of the pumping power is transferred to the BSWP mode. From the shift of the exciton
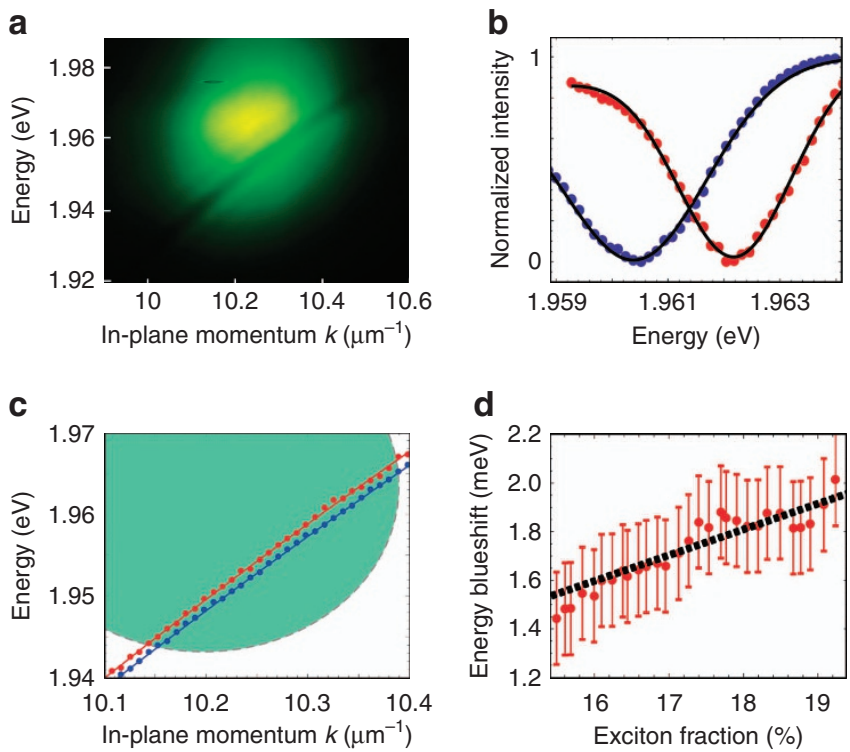

Figure 4 BSWP nonlinearities. (a) Bare experimental data of the BSWP dispersion in reflectance configuration (i.e., with resonant excitation of polaritons) at $150-\mu \mathrm{J} \mathrm{cm}^{-2}$ pumping energy. (b) Energy-resolved signal at inplane wavevector $k=10.34 \mu \mathrm{m}^{-1}$ under $150-\mu \mathrm{J} \mathrm{cm}^{-2}$ and $10-\mathrm{mJ} \mathrm{cm}^{-2}$ excitation energy densities (blue and red dots, respectively, with Gaussian fit as solid lines), showing the blueshift of the BSWP resonance. (c) Dots are the experimental BSWP dispersions with $150-\mu \mathrm{J} \mathrm{cm}^{-2}$ (blue) and $10-\mathrm{mJ} \mathrm{cm}^{-2}$ (red) resonant pump pulse. TMM calculations (solid lines) of the BSWP dispersion perfectly match the experimental values when considering an exciton energy of $2.13 \mathrm{eV}$ (blue) and a blue-shifted ( $8 \mathrm{meV}$ ) exciton (red). The green ellipse depicts the full width at half maximum of the laser spot in energy-momentum space. (d) The expected blueshift as a function of the excitonic fraction (black dashed line) fits the experimental results (red dots) for an exciton blueshift of $8 \mathrm{meV}$. 
transition, we can therefore estimate the interaction constant to be of the order of $10^{-3} \mu \mathrm{eV} \mu \mathrm{m}^{2}$, in accordance with the value indirectly extracted via non-resonant excitation of organic polaritons in a planar microcavity ${ }^{26}$. Thus, the strong coupling with organic molecules contributes to the enhancement of the nonlinear behavior of the BSW; nevertheless, despite the cryogenic temperatures, the nonlinearities offered by inorganic polaritons, are still between three and four orders of magnitude higher ${ }^{13,54}$.

\section{CONCLUSIONS}

Room temperature ballistic propagation of light-matter excitations in an organic semiconductor for distances well beyond one hundred microns is demonstrated thanks to the adoption of a BSW mode. Indeed, the BSWP speed exceeds $150 \mu \mathrm{m} \mathrm{ps}^{-1}$, which is two orders of magnitude higher than typical polariton velocities in standard planar microcavities. Moreover, through the resonant injection of a traveling polariton wave-packet, the exciton component of the BSWP manifests in the density-dependent self-energy of the system, showing blueshifts of the polariton resonance for increasing pump powers. This is the first direct measurement of this term, which arises from the excitonic components of polaritons, that enables tuning of the polariton energy and demonstrates a fundamental element for the implementation of polariton on-chip devices.

Although the interaction constant values reported for inorganic microcavities are higher than those observed in this work with organic semiconductors, these results are, however, very promising and can be further improved with the higher confinement of the electromagnetic field. In this respect, one of the most promising aspects of BSWP is that the surface can be easily patterned to localize the field and enhance the nonlinear optical response of organic materials, paving the way for the realization of ultrafast, low-loss polariton devices operating at room temperature.

\section{CONFLICT OF INTEREST}

The authors declare no conflict of interest.

\section{ACKNOWLEDGEMENTS}

GL is grateful to Gianluca Latini for the encouragement at the initial stage of his research path. This work was funded by the MIUR project Beyond Nano and the ERC project POLAFLOW (Grant No. 308136).

1 Kavokin A, Baumberg JJ, Malpuech G, Laussy FP. Microcavities. Oxford: OUP; 2007.

2 Bramati A, Modugno M. Physics of Quantum Fluids-New Trends and Hot Topics in Atomic and Polariton Condensates. Berlin: Springer; 2013.

3 Sanvitto D, Timofeev V. Exciton Polaritons in Microcavities-New Frontiers. Berlin: Springer; 2012.

4 Weisbuch C, Nishioka M, Ishikawa A, Arakawa Y. Observation of the coupled excitonphoton mode splitting in a semiconductor quantum microcavity. Phys Rev Lett 1992; 69: 3314-3317

5 Whittaker DM, Kinsler P, Fisher TA, Skolnick MS, Armitage A et al. Motional narrowing in semiconductor microcavities. Phys Rev Lett 1996; 77: 4792-4795.

6 Khitrova G, Gibbs HM, Kira M, Koch SW, Scherer A. Vacuum Rabi splitting in semiconductors. Nat Phys 2006; 2: 81-90.

7 Tassone $F$, Yamamoto $Y$. Exciton-exciton scattering dynamics in a semiconductor microcavity and stimulated scattering into polaritons. Phys Rev B 1999; 59: 10830-10842.

8 Savvidis PG, Baumberg JJ, Stevenson RM, Skolnick MS, Whittaker DM et al. Angleresonant stimulated polariton amplifier. Phys Rev Lett 2000; 84: 1547-1550.

9 Deng H, Weihs G, Santori C, Bloch J, Yamamoto Y. Condensation of semiconductor microcavity exciton polaritons. Science 2002; 298: 199-202.

10 Kasprzak J, Richard M, Kundermann S, Baas A, Jeambrun P et al. Bose-Einstein condensation of exciton polaritons. Nature 2006; 443: 409-414.

11 Balili R, Hartwell V, Snoke D, Pfeiffer L, West K. Bose-einstein condensation of microcavity polaritons in a trap. Science 2007; 316: 1007-1010.
12 Amo A, Sanvitto D, Laussy FP, Ballarini D, del Valle E et al. Collective fluid dynamics of a polariton condensate in a semiconductor microcavity. Nature 2009; 457 291-295.

13 Amo A, Lefrère J, Pigeon S, Adrados C, Ciuti C et al. Superfluidity of polaritons in semiconductor microcavities. Nat Phys 2009; 5: 805-810.

14 Lagoudakis KG, Wouters M, Richard M, Baas A, Carusotto I et al. Quantized vortices in an exciton-polariton condensate. Nat Phys 2008; 4: 706-710.

15 Lagoudakis KG, Ostatnicky T, Kavokin AV, Rubo YG, Andre R et al. Observation of half-quantum vortices in an exciton-polariton condensate. Science 2009; 326: 974-976.

16 Sanvitto D, Marchetti FM, Szymańska MH, Tosi G, Baudisch M et al. Persistent currents and quantized vortices in a polariton superfluid. Nat Phys 2010; 6: 527-533.

17 De Giorgi M, Ballarini D, Cancellieri E, Marchetti FM, Szymanska MH et al. Control and ultrafast dynamics of a two-fluid polariton switch. Phys Rev Lett 2012; 109: 266407.

18 Marsault F, Nguyen HS, Tanese D, Lemaître A, Galopin E et al. Realization of an all optical exciton-polariton router. App/ Phys Lett 2015; 107: 201115.

19 Cerna R, Léger Y, Paraïso TK, Wouters M, Morier-Genoud F et al. Ultrafast tristable spin memory of a coherent polariton gas. Nat Commun 2013; 4: 2008.

20 Sich M, Krizhanovskii DN, Skolnick MS, Gorbach AV, Hartley R et al. Observation of bright polariton solitons in a semiconductor microcavity. Nat Photon 2012; 6 : 50-55.

21 Snoke D. Microcavity polaritons: A new type of light switch. Nat Nanotechnol 2013; 8: 393-395.

22 Liew TCH, Shelykh IA, Malpuech G. Polaritonic devices. Phys E Low Dimens Syst Nanostructures 2011; 43: 1543-1568.

23 Miller DAB. Are optical transistors the logical next step? Nat Photon 2010; 4: 3-5.

24 Lidzey DG, Bradley DDC, Skolnick MS, Virgili T, Walker S et al. Strong exciton-photon coupling in an organic semiconductor microcavity. Nature 1998; 395: 53-55.

25 Agranovich VM, Litinskaia M, Lidzey DG. Cavity polaritons in microcavities containing disordered organic semiconductors. Phys Rev B 2003; 67: 085311.

26 Daskalakis KS, Maier SA, Murray R, Kéna-Cohen S. Nonlinear interactions in an organic polariton condensate. Nat Mater 2014; 13: 271-278.

27 Plumhof JD, Stöferle T, Mai L, Scherf U, Mahrt RF. Room-temperature Bose-Einstein condensation of cavity exciton-polaritons in a polymer. Nat Mater 2014; 13: 247-252.

28 Ballarini D, De Giorgi M, Cancellieri E, Houdré R, Giacobino E et al. All-optical polariton transistor. Nat Commun 2013; 4: 1778.

29 Antón C, Liew TCH, Tosi G, Martín MD, Gao T et al. Dynamics of a polariton condensate transistor switch. App/ Phys Lett 2012; 101: 261116.

30 Liew TCH, Kavokin AV, Ostatnický T, Kaliteevski M, Shelykh IA et al. Exciton-polariton integrated circuits. Phys Rev B 2010; 82: 033302.

31 Nguyen HS, Vishnevsky D, Sturm C, Tanese D, Solnyshkov D et al. Realization of a double-barrier resonant tunneling diode for cavity polaritons. Phys Rev Lett 2013; 110: 236601.

32 Descrovi E, Sfez T, Dominici L, Nakagawa W, Michelotti F et al. Near-field imaging of Bloch surface waves on silicon nitride one-dimensional photonic crystals. Opt Express 2008; 16: 5453-5464.

33 Descrovi E, Sfez T, Quaglio M, Brunazzo D, Dominici L et al. Guided bloch surface waves on ultrathin polymeric ridges. Nano Lett 2010; 10: 2087-2091.

34 Lerario G, Cannavale A, Ballarini D, Dominici L, De Giorgi M et al. Room temperature Bloch surface wave polaritons. Opt Lett 2014; 39: 2068-2071.

35 Aberra Guebrou S, Symonds C, Homeyer E, Plenet JC, Gartstein YN et al. Coherent emission from a disordered organic semiconductor induced by strong coupling with surface plasmons. Phys Rev Lett 2012; 108: 066401.

36 Yu LB, Barakat E, Sfez T, Hvozdara L, Di Francesco J et al. Manipulating Bloch surface waves in 2D: a platform concept-based flat lens. Light Sci Appl 2014; 3: e124, doi:10.1038/Isa.2014.5.

37 Angelini A, Lamberti A, Ricciardi S, Frascella F, Munzert P et al. In-plane 2D focusing of surface waves by ultrathin refractive structures. Opt Lett 2014; 39: 6391-6394.

38 Angelini A, Barakat E, Munzert P, Boarino L, De Leo N et al. Focusing and extraction of light mediated by Bloch surface waves. Sci Rep 2014; 4: 5428.

39 Yu LB, Barakat E, Di Francesco J, Herzig HP. Two-dimensional polymer grating and prism on Bloch surface waves platform. Opt Express 2015; 23: 31640-31647.

40 Ballarini M, Frascella F, Michelotti F, Digregorio G, Rivolo P et al. Bloch surface wavescontrolled emission of organic dyes grafted on a one-dimensional photonic crystal. App/ Phys Lett 2011; 99: 043302.

41 Ballarini M, Frascella F, Enrico E, Mandracci P, De Leo N et al. Bloch surface wavescontrolled fluorescence emission: coupling into nanometer-sized polymeric waveguides. Appl Phys Lett 2012; 100: 063305.

42 Liscidini M, Gerace D, Sanvitto D, Bajoni D. Guided Bloch surface wave polaritons. App/ Phys Lett 2011; 98: 121118.

43 Sinibaldi A, Rizzo R, Figliozzi G, Descrovi E, Danz N et al. A full ellipsometric approach to optical sensing with Bloch surface waves on photonic crystals. Opt Express 2013; 21: 23331-23344.

44 Feist J, Garcia-Vidal FJ. Extraordinary exciton conductance induced by strong coupling. Phys Rev Lett 2015; 114: 196402.

45 Schachenmayer J, Genes C, Tignone E, Pupillo G. Cavity-enhanced transport of excitons. Phys Rev Lett 2015; 114: 196403.

46 Leggio B, Messina R, Antezza M. Thermally activated nonlocal amplification in quantum energy transport. Europhys Lett 2015; 110: 40002. 
47 Orgiu E, George J, Hutchison JA, Devaux E, Dayen JF et al. Conductivity in organic semiconductors hybridized with the vacuum field. Nat Mater 2015; 14: 1123-1129.

48 Ballarini D, De Giorgi M, Gambino S, Lerario G, Mazzeo M et al. Polariton-induced enhanced emission from an organic dye under the strong coupling regime. Adv Opt Mater 2014; 2: 1076-1081.

49 Coles DM, Michetti P, Clark C, Tsoi WC, Adawi AM et al. Vibrationally assisted polaritonrelaxation processes in strongly coupled organic-semiconductor microcavities. Adv Funct Mater 2011; 21: 3691-3696.

50 Michetti P, La Rocca GC. Exciton-phonon scattering and photoexcitation dynamics in J-aggregate microcavities. Phys Rev B 2009; 79: 035325.

51 Steger M, Gautham C, Snoke DW, Pfeiffer L, West K. Slow reflection and two-photon generation of microcavity exciton-polaritons. Optica 2015; 2: 1-5.

52 Nelsen B, Liu GQ, Steger M, Snoke DW, Balili R et al. Dissipationless flow and sharp threshold of a polariton condensate with long lifetime. Phys Rev $X$ 2013; 3: 041015.
53 Wertz E, Ferrier L, Solnyshkov DD, Johne R, Sanvitto D et al. Spontaneous formation and optical manipulation of extended polariton condensates. Nat Phys 2010; 6: 860-864.

54 Vladimirova M, Cronenberger S, Scalbert D, Kavokin KV, Miard A et al. Polaritonpolariton interaction constants in microcavities. Phys Rev B 2010; 82: 075301.

(c) (i) (2) This work is licensed under a Creative Commons Attributioncc) NonCommercial-ShareAlike 4.0 International License. The images or other third party material in this article are included in the article's Creative Commons license, unless indicated otherwise in the credit line; if the material is not included under the Creative Commons license, users will need to obtain permission from the license

holder to reproduce the material. To view a copy of this license, visit http:// creativecommons.org/licenses/by-nc-sa/4.0/

(C) The Author(s) 2017

Supplementary Information for this article can be found on the Light: Science \& Applications' website (http://www.nature.com/lsa). 